\title{
Screening of guava (Psidium guajava) leaves extracts against $\beta$-hematin formation
}

\begin{abstract}
Background: Malaria control is crucial as a result of the growing antimalarial drugresistance not only in South-East Asia but also in Africa. Developing novel, safe, effective, and affordable drugs is a key to combat malaria. Medicinal plants and some fruits are intensive sources in the drug discovery effort to treat malaria

Objective: The main aim of the work is to investigate Guava (Psidium guajava) infused water and water-ethanol leaf extracts against $\beta$-hematin formation in-vitro.

Methodology: To determine the identity of any possible active secondary metabolite in $P$. guajava leaves, a full scan LC-ESi-MS-PDA was run within a mass range of 100-1200 amu. Moreover, the in-vitro colorimetric semi-quantitative assays that are based on $\beta$-hematin inhibition and optical density measurement at $405 \mathrm{~nm}$ were utilized.

Results and discussion: The effect of using $35 \%$ ethanol in extracting guava leaves showed that concentrations between 1 and $0.4 \mathrm{mg} / \mathrm{ml}$ have an activity superior to guava water extract and comparable to the positive control. Among the identified flavonoids glycosides are quercetin 3-O-glycoside, quercetin-galloylhexoside, morin-3-O-lyxoside, morin-3-Oarabinoside, quercein-3-O-arabinoside (guaijaverin) and ellagic acid. This indicates to an encouraging complexation with the free heme over $\beta$-hematin formation.
\end{abstract}

Keywords: Guava, Psidium guajava, LC-MS, antimalaria, quercetin-3-O-glycoside, quercitrin, $\beta$-hematin
Volume 9 Issue | - 202|

\author{
Mutaz Akkawi,' Saleh Abu-Lafi, ${ }^{2}$ Qassem \\ Abu-Remeleh, ${ }^{2}$ Pierre Lutgen ${ }^{3}$ \\ 'Life Sciences Department, College of Science and Technology, \\ Al-Quds University, Palestine \\ ${ }^{2}$ Faculty of Pharmacy,Al-Quds University, Palestine \\ ${ }^{3}$ IFBV-BELHERB, Luxembourg
}

Correspondence: Saleh Abu-Lafi: Faculty of Pharmacy,Al-Quds University, West Bank, Palestine, Tel + +972-2-2799360,

Email sabulafi@staff.alqud.edu

Received: January 23, 2021 | Published: February 02, 202 |

\section{Introduction}

Malaria is a deadly disease caused by a protozoan parasite which belongs to Plasmodium genus that spreads to humans through the bites of infected female Anopheles mosquitoes. ${ }^{1}$ Although there are different species of Plasmodium causing malaria, Plasmodium falciparum, is considered the deadliest one. ${ }^{1}$ Approximately, quarter of a million new cases were reported in 2019 of which 409,000 died because of malaria. ${ }^{1}$ The prevalence of malaria endemic is among the poorest counties, mainly in Africa, and about two-thirds of deaths are among children under the age of five. ${ }^{1}$ Fortunately, malaria is preventable and treatable. During the trophozoite stage of the parasite intraerythrocytic life cycle, Plasmodium parasites use special enzymes to digest hemoglobin in the acidic food vacuole leading to the formation of monomeric heme units called ferriheme or ferriprotoporphyrin (IX). However, the parasites cannot tolerate the toxicity of these free heme. ${ }^{2}$ Therefore, it converts heme rapidly into an inert, non-toxic polymer called hemozoin. ${ }^{3}$ The synthetic version of hemozoin is called $\beta$-hematin, usually utilized in biological assay determination. Antimalarial drugs inhibit hemozoin formation and then lead to the death of the parasite. ${ }^{2,3}$

As a result of the growing antimalarial drug-resistance, the number of deaths is on the rise. Therefore, developing new, safe, effective and affordable hemozoin inhibitors is indispensable to combat malaria. The use of natural products with therapeutic properties is as ancient as human civilization. Medicinal plants have been used in the treatment and improvement of human diseases including malaria. ${ }^{4,5}$ For example, the leaves of Psidium guajava (Myrtaceae), commonly known as guava is used for the treatment of malaria in Nigeria. ${ }^{6,7}$ Besides documenting the safety of guava, it is also reported about its benefits against many other conditions including gastrointestinal infections, respiratory infections, oral/dental infections, skin infections, diabetes, cardiovascular/hypertension, cancer, malnutrition, women problems, pain, fever, liver problems and kidney problems. ${ }^{8}$
Guava leaves comprise diverse phenolic compounds and flavonoids. The main active substances in guava leaves are gallic acid, caffeic acid, flavonoids, guaijaverin, tannins, carotenoids, and triterpenoids. ${ }^{9-11}$ Moreover, guava leaf oil using gas chromatography revealed a mixture of sesquiterpene hydrocarbons, oxygenated sesquiterpenes..$^{12}$ Recently, scanning of all the polar constituents mainly phenolic compounds present in guava leaves extract were extensively identified using HPLC-ESI-MS. ${ }^{13,14}$

The present work aimed to determine the in-vitro inhibitory influence of guava leaves infusion water and ethanol extracts on the $\beta$-hematin formation using a semi-quantitative screening method. Moreover, the principal polar secondary metabolites in guava leaves that are extracted out of the infusion process are scanned and identified in an effort to discover novel antimalarial leads.

\section{Materials and methods}

Acetonitrile $(\mathrm{ACN})$ and ultra-pure water $\left(\mathrm{H}_{2} \mathrm{O}\right.$, prepared by using a Millipore Milli-Q plus water purification system), formic acid and ammonium formate were all of LC-MS grades. Dimethyl sulfoxide (DMSO), chloroquine diphosphate salt (CQ), morin, quercetin, catechin and quercitrin hydrate analytical standards, sodium acetate, and hemin chloride were all purchased from Sigma-Aldrich. Glacial acetic acid was purchased from Fluka. Ethanol (EtOH) was from Merck. Guava leaves (Psidium guajava) was collected in 2020 from Qalqiliya city at northern part of Palestine. The leaves dried under shade and stored at in amber bottle and kept in the fridge until used.

\section{Preparation of guava leaves infusion}

\section{Preparation of the guava extracts-Method A}

Air-dried guava (P. guajava) leaves were grinded into coarse powder; extracted by soaking 2-grams in $150 \mathrm{ml}$ of distilled hot water at $90^{\circ} \mathrm{C}$ for 20 minutes. Then it is left to cool down at room 
temperature, followed by filtration using MN $615 \varnothing 110 \mathrm{~mm}$ filter paper. The water was evaporated by heating at $60-70^{\circ} \mathrm{C}$ under reduced pressure using a rotary evaporator (IKA WEREKRV06-ML), followed by freeze-drying (Labconco freeze drier) until a constant weight was achieved. The final dried extract was stored in opaque bottles and kept in desiccators until analysis by LC-MS.

\section{Extraction of the guava extracts-Method B}

Air-dried guava leaves (P. guajava) were grinded into coarse powder; extracted by soaking $(1: 10, \mathrm{wt} / \mathrm{vol})$ of dried plant leaves in $35 \%$ ethanol, left for about 24 hours at room temperature. The extract was then filtered using Whatman filter paper (No 42). The crude aqueous ethanol extract was evaporated first using rotary evaporator at $60-80^{\circ} \mathrm{C}$ under reduced pressure, followed by lyophilization until constant weight was obtained, then stored at $-20^{\circ} \mathrm{C}$ until LC-MS analysis.

\section{Semi-quantitative in-vitro method}

The method used was as described by Deharo et al., ${ }^{15}$ semiquantitative micro-assay. The details of the assay have been described in our previous publication with some modification. ${ }^{16}$ In brief, a mixture containing $50 \mu \mathrm{L}$ of $0.5 \mathrm{mg} / \mathrm{mL}$ hemin chloride freshly dissolved in DMSO, $100 \mu \mathrm{L}$ of $0.5 \mathrm{M}$ sodium acetate buffer $(\mathrm{pH}$ 4.4) and $50 \mu \mathrm{L}$ of the standard solution or either the negative (water, alkaline water, DMSO) or positive (chloroquine or amodiaquine) controls, was incubated in a non-sterile 96-well flat bottom plate at $37^{\circ} \mathrm{C}$ for $18-24$ hours. The solutions were added to the plate in the above order. The plate was then centrifuged for $10 \mathrm{~min}$ at 4000 $\mathrm{rpm}$. The supernatant was removed and the $\mathrm{pH}$ of the reaction was measured. The final $\mathrm{pH}$ of the mixture was between (5.0-5.2). The wells were washed with $200 \mu \mathrm{L}$ DMSO per well to remove unreacted hemin. The plate was centrifuged again followed by discharging the supernatant. The $\beta$-hematin remaining was then dissolved in $200 \mu \mathrm{L}$ of $0.1 \mathrm{M} \mathrm{NaOH}$ to form an alkaline hematin that can be measured spectrophotometrically at $405 \mathrm{~nm}$ using ELISA reader. Positive controls were dissolved in ultra-pure water for chloroquine and in DMSO for amodiaquine.

\section{Instrumentation}

The chromatographic column used was an Acquity UPLC BEH C18 (50mm x 2.1 mm I.D., $1.7 \mu \mathrm{m})$ connected to an Acquity BEH C18 $1.7 \mu \mathrm{m}$ guard column (Vanguard $2.1 \times 5 \mathrm{~mm}$ ) from Waters, Milford, MA, USA). All the analyses were accomplished using Waters Acquity UPLC H-Class system (Waters, Milford, MA, USA) equipped with a binary solvent manager, sampler manager, column manager, Acquity QDa detector, photodiode array detector (PDA) and Empower3 software.

\section{Chromatographic conditions}

The LC-MS column and sample temperature were maintained at $35^{\circ} \mathrm{C}$ and $25^{\circ} \mathrm{C}$ respectively. The mobile phase consisted of 0.1 $\%$ formic acid in water (A) and $0.1 \%$ formic acid in acetonitrile (B) at a constant flow rate of $0.4 \mathrm{~mL} / \mathrm{min}$. The linear gradient starting conditions was $98 \%$ A, increased to $100 \%$ B over 12 minutes. Then it stayed at $100 \%$ B for another two minutes. Prior any injection, the mobile phase was equilibrated for 5 minutes. The diluent was $100 \%$ ethanol and the crude guava sample was sonicated for 5 minutes and filtered prior to $2 \mu \mathrm{L}$ injection. The detection range of the PDA was from $210-500 \mathrm{~nm}$. The Acquity QDa ionization modes were in the negative and positive ESi and the mass range was between 100-1200 Da.

\section{Results and discussion}

In the literature, it was stated that guava ( $P$. guajava) leaves comprise many flavonoids such as morin, quercetin, other related compounds such as morin-3-O-lyxopyranoside and quercetin3-O-arabinoside. ${ }^{17,18}$ These compounds were isolated and fully characterized using chemical and spectroscopic techniques. ${ }^{17}$ Recently, we investigated the in-vitro inhibitory effect of some pure individual flavonoid compounds such as morin, quercitrin and quercetin to which they all showed promising antimalarial activity when compared to chloroquine and amodiaquine antimalarial drugs. ${ }^{16}$ We thought it would be advantageous to investigate guava leaves extracts which are evidently a rich source of such flavonoids. Therefore, water and water-ethanol $(65: 35 ; \mathrm{v}: \mathrm{v})$ extracts of guava leaves were prepared by infusion and soaking respectively and directly chromatographed afterwards by LC-ESi-MS-machine. The in-vitro inhibitory influence on $\beta$-hematin formation using the semi-quantitative screening method was examined. Figure 1 shows the chemical structures of the flavonoids identified in the water and water-ethanol extracts.

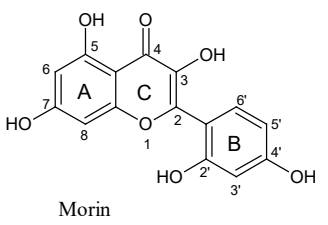

(c)

Quercitrin

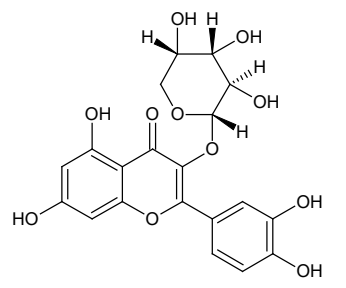

Quercein-3-O-arabinoside (guaijaverin)<smiles>O=c1c(O)c(-c2ccc(O)c(O)c2)oc2cc(O)cc(O)c12</smiles>

Quercetin

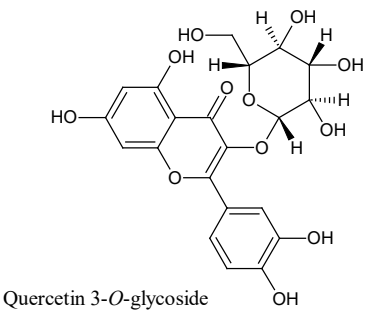

Ellagic acid

Figure I Chemical structures of some identified flavonoids in guava leaves by infusion.

Figures 2-5 show the chromatograms of quercitrin, quercetin, guava extract in water and guava extract in $35 \%$ ethanol respectively at a monitoring wavelength of $350 \mathrm{~nm}$, the maximum absorption wavelength of most of flavonoids present in guava. As can be seen in figure 2, quercitrin standard eluted at 4.018 minutes and its UVVis spectrum showed two maxima at 255.5 and $348.9 \mathrm{~nm}$ respectively. Quercitrin mass spectrum in the negative ESi mode shows $\mathrm{m} / \mathrm{z}$ of 447 amu corresponding to typical deprotonated pseudo molecular ions [M-1] of quercitrin having a molecular mass of $448 \mathrm{amu}$. The peak at $895 \mathrm{amu}$ represents the dimer adduct of quercitrin at $\mathrm{m} / \mathrm{z}$ of [2M$1]$, seen apparently because of the relatively high concentration of the injected standard. Quercetin however eluted later at 5.9 minutes and its UV-Vis spectrum showed two maxima at 254.3 and $348.2 \mathrm{~nm}$ respectively (figure 3). Quercetin mass spectrum in the negative ESi 
mode shows $\mathrm{m} / \mathrm{z}$ of $301 \mathrm{amu}$ corresponding to typical deprotonated pseudo molecular ions $[\mathrm{M}-1]^{-}$of morin having a molecular mass of $302 \mathrm{amu}$. The peak at $603 \mathrm{amu}$ represents the dimer adduct of quercitrin at $\mathrm{m} / \mathrm{z}$ of [2M-1] (figure 3 ). When the water guava leaves infusion extract was injected, quercitrin was seen at 4.018 minutes while quercetin was below the detection limit (figure 4). The same was observed in the 35\% ethanol extract (figure 5). Extraction methods based on soxhlet, sonication, accelerated solvent extraction and microwave-assisted extraction are relatively harsh in comparison to infusion or soaking methods. In the last decade, we deliberately utilized water infusion as a method of choice to imitate tea preparation that everybody could make in the kitchen to treat malaria. The use of water as a solvent explains the early elution of polar glycoside flavonoids. For example, the major peaks that emerged at 3.01, $3.17,3.43,3.58,3.76$ minutes showed nominal masses of 463, $463,433,433,433$ amu respectively using negative Esi-MS mode (figure 4). The first two isomeric peaks most (deprotonated pseudo molecular ions [M-1] of $463 \mathrm{amu}$ ) probably correspond to quercetin 3-O-glycoside and quercetin-galloylhexoside isomers that were isolated and identified previously using 90\% aqueous methanol. ${ }^{17}$ Moreover, the other three isomers sharing the molecular mass of 434 amu (deprotonated isomers pseudo molecular ions [M-1] of $463 \mathrm{amu}$ ) are indicative of same isomers that have been systematically isolated and identified as morin-3-O-lyxoside, morin-3-O-arabinoside and quercein-3-O-arabinoside (guaijaverin) respectively using LC-APCIMS, NMR spectroscopic techniques and acid hydrolysis. ${ }^{18}$ All these isomeric compounds designate to the presence of quercetin or morin aglycone based moiety after losing their glycones (sugar portion). At retention 2.6 mins the peak molecular weight is 301 amu probably indicative of ellagic acid (molecular mass $302 \mathrm{amu}$ ). The small two peaks eluted at 3.88 and 4.7 minutes shows [M-1] of 543 and $585 \mathrm{amu}$ revealing guavinoside $\mathrm{A}$ isomer and guavinoside $\mathrm{C}$ respectively while the peak at 6.6 minutes is identified as 2,6-dihydroxy-3-methyl-4-O(6"-O-galloyl- $\beta$-Dglucopyranosyl)-benzophenone. ${ }^{14}$

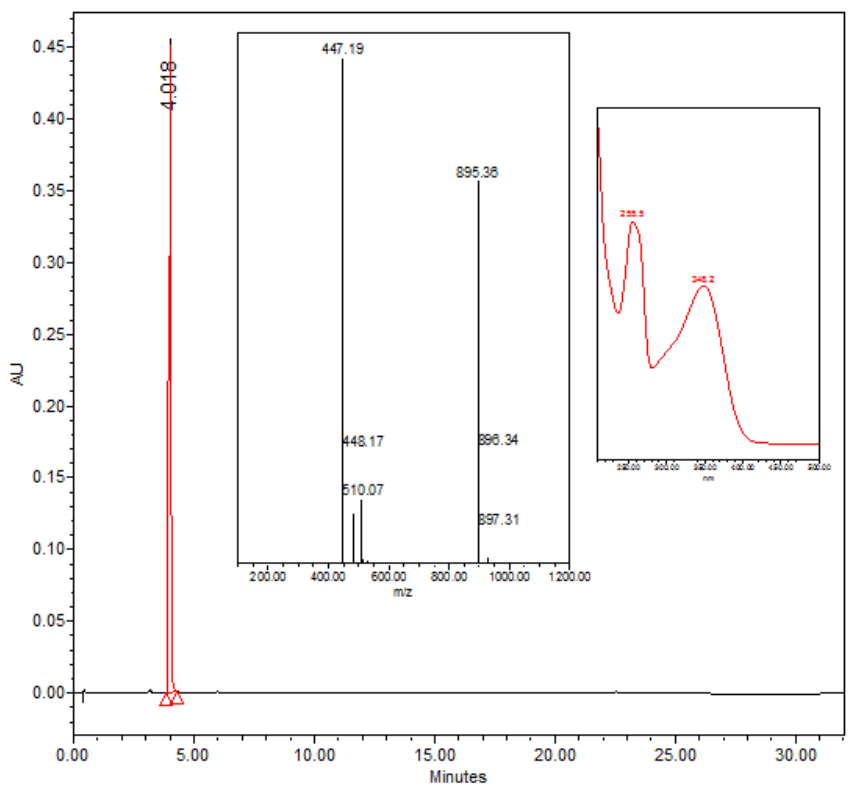

Figure 2 LC-PDA-MS of quercitrin standard.

\section{$\beta$-hematin inhibition}

There are semi-quantitative assays that have been developed to test for in-vitro $\beta$-hematin inhibition. The $\beta$-hematin is the synthetic analog of hemozoin and it has been confirmed to be similar chemically, spectroscopically and crystallographically. ${ }^{15}$ In our previous work, we noticed an encouraging potential of flavonoids such as morin, quercetin, quercitrin as $\beta$-hematin inhibitors. ${ }^{16}$ The inhibitory effect of guava leaves water and water-ethanolic extracts on the biomineralization of ferriprotoporphyrin IX to $\beta$-hematin were investigated. The potential antimalarial efficiency was assessed by measurement of the absorption of $\beta$-haematin dye at $405 \mathrm{~nm}$ in comparison to water $\left(\mathrm{H}_{2} \mathrm{O}\right)$ negative control, chloroquine (CQ- $0.1 \mathrm{mg} /$ $\mathrm{ml}$ ) positive control. Results are shown in figures 6-8. Absorption is inversely proportional to extract efficiency a while it is directly proportional to $\beta$-hematin content, i.e., the lower the absorption, the more efficient the extract and the stronger the inhibitory effect towards $\beta$-haematin formation.

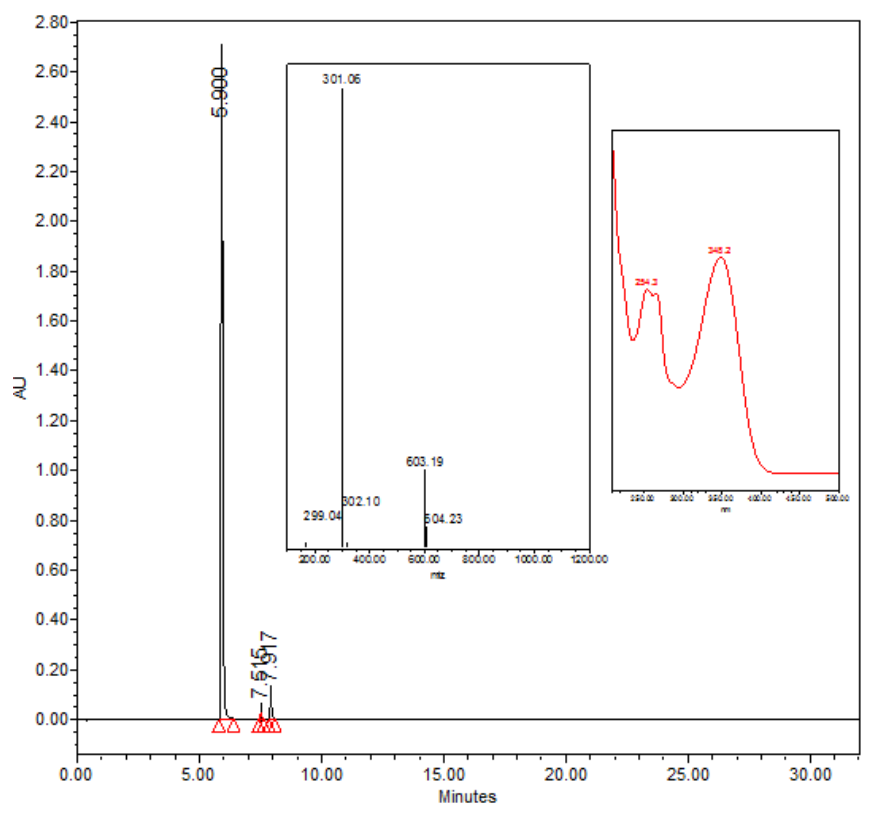

Figure 3 LC-PDA-MS of quercetin standard.

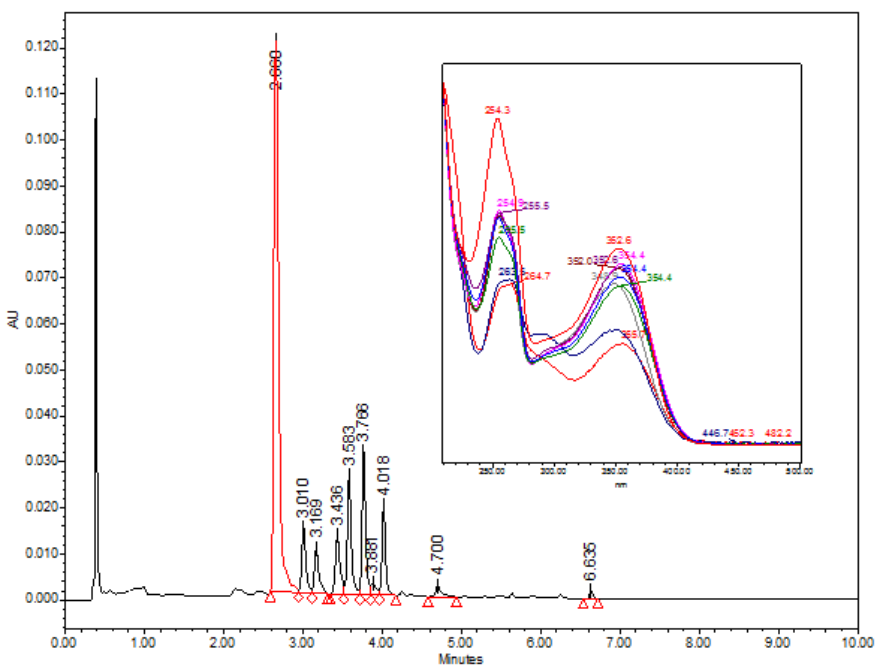

Figure 4 Chromatogram using LC-PDA-MS of crude guava leaves extract from water infusion.

As shown in figure 6, guava leaves water extract significantly inhibited the $\beta$-hematin formation at $100 \%$ concentrated filtrate (method A, prior lyophilization) up to $30 \%$ dilutions with a notably 
lesser activity at $20 \%$ dilution. The $10 \%$ diluted solution did not show any significant effect on the in-vitro $\beta$-hematin inhibition giving rise to a very high absorption value at $405 \mathrm{~nm}$. Directly afterward, the test was continued using the accurately weighted lyophilized guava water extract (method A, post lyophilization) starting from $1 \mathrm{mg} / \mathrm{ml}$ up to $0.1 \mathrm{mg} / \mathrm{ml}$ dilutions. Figure 7 shows a moderate activity in the range between 1 and $0.5 \mathrm{mg} / \mathrm{ml}$ and pretty less at $0.4-0.3 \mathrm{mg} / \mathrm{ml}$ but no activity after $0.2 \mathrm{mg} / \mathrm{ml}$

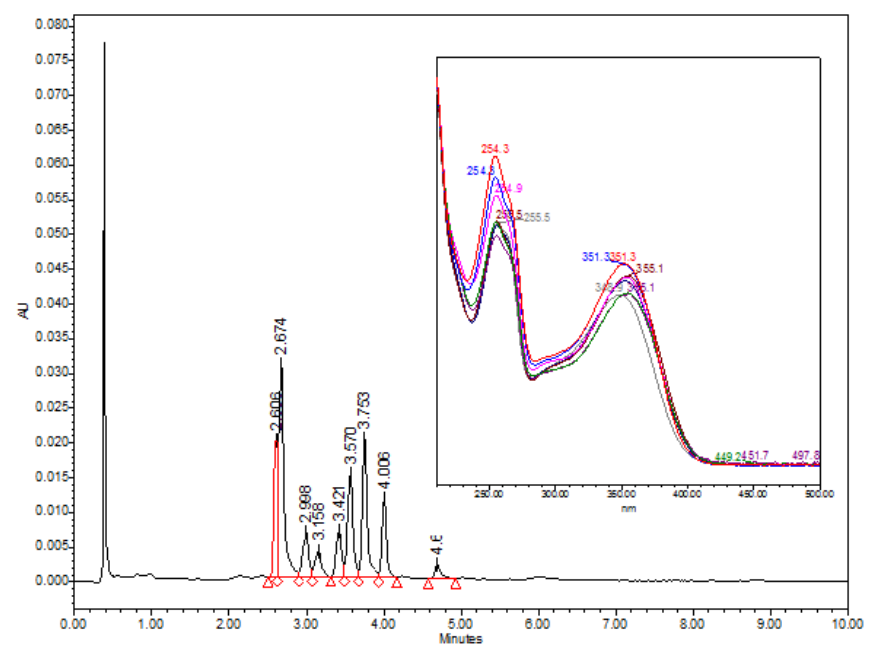

Figure 5 Chromatogram using LC-PDA-MS of crude guava leaves extract from $35 \% \mathrm{EtOH}$.

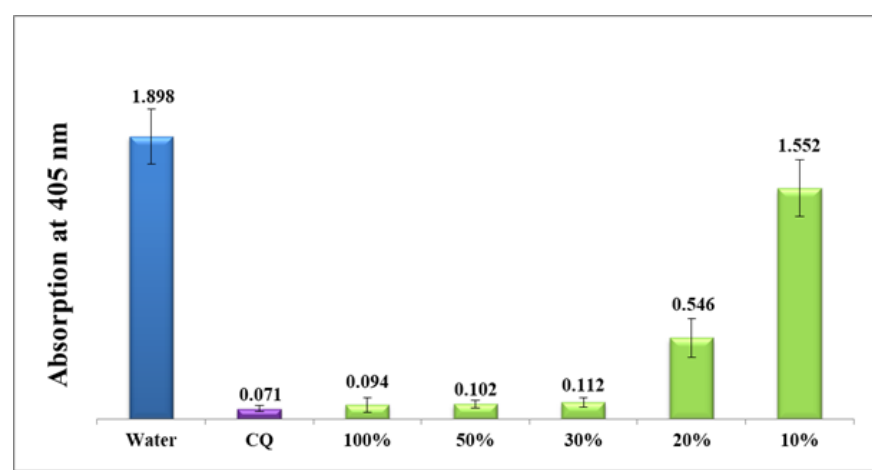

Figure 6 Column diagram of the absorption at $405 \mathrm{~nm}$ of guava leaves water extract (Method A) at different dilutions using distilled water in comparison to water negative control and $(\mathrm{CQ}-0.1 \mathrm{mg} / \mathrm{ml})$ positive control. Each result represents an average of 16 experiments.

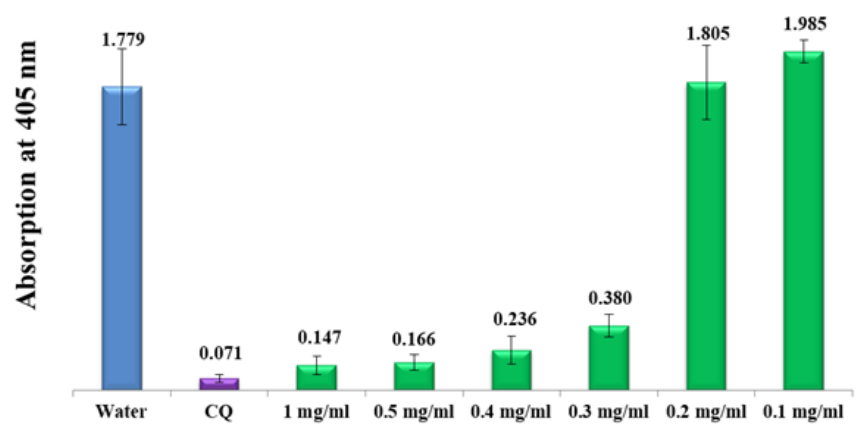

Figure 7 Efficiency of lyophilized guava crude water extract infusion (Method A) at different dilutions compared to water negative control and CQ-0.I $\mathrm{mg} / \mathrm{ml}$ positive control. Each result represents the average of 16 individual experiments.
The effect of using a binary solvent mixture (35\% ethanol) in extracting guava leaves compounds is shown in figure 8 . At concentrations between 1 and $0.4 \mathrm{mg} / \mathrm{ml}$, the activity was superior to guava water extract and comparable to the positive control. However, at $0.2 \mathrm{mg} / \mathrm{ml}$ level and above, the activity was virtually lost.

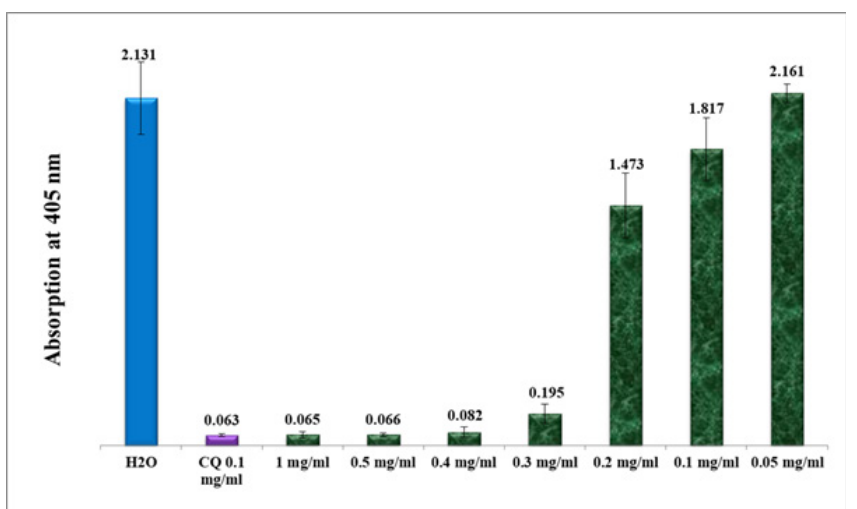

Figure 8 Column diagram representing antimalarial efficiency (absorption of dissolved $\beta$-hematin at $405 \mathrm{~nm}$ ) of $35 \%$ ethanol extracts of guava leaves (Method-B) in comparison to negative water control and positive control $(\mathrm{CQ}-0.1 \mathrm{mg} / \mathrm{ml})$. Each result represents an average of 16 individual experiments.

While it has been proven previously and through the experimental results of the current study that flavonoids and flavonoids glycosides molecules alike inhibit hemozoin formation, the exact mechanism of how it happens is not fully understood. It is assumed that the mechanism happens through the development of a stable complex comprising the antimalarial drug and ferriheme. ${ }^{16}$ The driving force of such a stable complexes are combined of the formation of hydrogen bonding between free propionic carboxylate heme and the hydroxychromone of the flavonoids ring B and C. Further, the hydrophobic $\pi-\pi$ stacking between flavonoid rings and porphyrin rings may also play a significant role in such a mechanism.

\section{Conclusion}

Guava leaves extract containing flavonoids and flavonoids glycosides showed promising antimalarial activity compared to chloroquine and amodiaquine positive controls in vitro. The effect of using $35 \%$ ethanol as extraction solvents of guava leaves provided superior activity in comparison to guava water extract and comparable to the positive control. Flavonoids glycosides also showed encouraging antimalarial activity by the formation of complex with free heme over $\beta$-hematin formation.

\section{Acknowledgments}

None.

\section{Conflicts of interest}

The authors declare that there is no conflict of interest.

\section{References}

1. World Malaria Report. World Health Organization; 2020 .

2. Kumar S, Guha M, Choubey V, et al. Antimalarial Drugs Inhibiting Hemozoin (Beta-Hematin) Formation: A Mechanistic Update. Life Sciences. 2007;80(9):813-828.

3. Goldberg D, Slater A, Cerami A, et al. Hemoglobin degradation in the malaria parasite Plasmodium falciparum: An ordered process in a unique organelle. Proc Natl Acad Sci USA. 1990;87(8):2931-2935. 
4. Seo J, Lee S, Elam ML, et al. Study to find the best extraction solvent for use with guava leaves (Psidium guajava L.) for high antioxidant efficacy. Food Sci Nutr. 2014;2(2):174-180.

5. Gutierrez RM, Mitchell S, Solis RV. Psidium guajava: a review of its traditional uses, phytochemistry and pharmacology. J. Ethnopharmacol. 2008;117(1):1-27.

6. Ukaga CN, Nwoke BEB, Onyeka PIK, et al. The use of herbs in malaria treatment in parts of Imo State, Nigeria. Tanzania Health Res Bull. 2006;8(3):183-185.

7. Rwang PG, Effoim OE, Mercy KP, et al. Effects of Psidium guajava (Guava) Extracts on Immature Stage of Mosquito. Int $J$ Complement Alt Med. 2016;4(5):00132.

8. Daswani P, Gholkar M, Birdi T. Psidium guajava: A Single Plant for Multiple Health Problems of Rural Indian Population. Pharmacogn Rev. 2017;11(22):167-174.

9. Okuda T, Yoshida T, Hatano T, et al. Guavins A, C and D, complex tannin from Psidium guajava. Chem Pharm Bull. 1987;35(1):443-446.

10. Mercadante AZ, Steck A, Pfander H. Carotenoids from Guava (Psidium guajava L.): isolation and structure elucidation. J Agric Food Chem. 1999;47(1):145-151.

11. Shao M, Wang Y, Huang XJ, et al. Four new triterpenoids from the leaves of Psidium guajava. J Asian Nat Prod Res. 2012;14(4):348-354.
12. Wang F, Chen YH, Zhang YJ, et al. Chemical Components and Bioactivities of Psidium guajava. Journal of Food Nutrition and Safety. 2014;5(2):98-114.

13. Díaz-de-Cerio E, Verardo V, Gómez-Caravaca A, et al. Determination of Polar Compounds in Guava Leaves Infusions and Ultrasound Aqueous Extract by HPLC-ESI-MS. Journal of Chemistry. 2015;2015:250919.

14. Elixabet Díaz-de-Cerio, Ana María Gómez-Caravaca, Vito Verardo, Alberto Fernández-Gutiérrez, et al. Determination of guava (Psidium guajava L.) leaf phenolic compounds using HPLC-DAD-QTOF-MS. Journal of Functional Foods. 2016;22:376-388.

15. Deharo E, Garcia RN, Oporto P, et al. A non-radiolabelled ferriprotoporphyrin biomineralisation inhibition test for the high throughput screening of antimalarial compounds. Exp Parasitol. 2002;100(4):252-256.

16. Abu-Lafi S, Akkawi M, Al-Rimawi F, et al. Morin, quercetin, catechin and quercitrin as novel natural antimalarial candidates. Pharm Pharmacol Int J. 2020;8(3):184-190.

17. Hidetoshi A, Gem-ichi D. Isolation of antimicrobial compounds from guava (Psidium guagava L.) and their structural elucidation. Biosci Biotechnol Biochem. 2002;66(8):1727-1730.

18. Rattanachaikunsopon P, Phumkhachorn P. Bacteriostatic effect of flavonoids isolated from leaves of Psidium guajava on fish pathogens. Fitoterapia. 2007;78(6):434-436. 